\title{
Grégoire et la cause des Noirs (1789 - 1831). Combats et projets
}

\section{Bernard Gainot}

\section{(2) OpenEdition \\ 1 Journals}

Édition électronique

URL : https://journals.openedition.org/ahrf/7223

DOI : 10.4000/ahrf.7223

ISSN : 1952-403X

\section{Éditeur :}

Armand Colin, Société des études robespierristes

\section{Édition imprimée}

Date de publication : 1 septembre 2006

Pagination : 181-185

ISSN : 0003-4436

\section{Référence électronique}

Bernard Gainot, "Grégoire et la cause des Noirs (1789 - 1831). Combats et projets », Annales

historiques de la Révolution française [En ligne], 345 | juillet-septembre 2006, mis en ligne le 08 juillet 2008, consulté le 01 juillet 2021. URL : http://journals.openedition.org/ahrf/7223 ; DOI : https:// doi.org/10.4000/ahrf.7223

Ce document a été généré automatiquement le 1 juillet 2021.

Tous droits réservés 


\title{
Grégoire et la cause des Noirs (1789 - 1831). Combats et projets
}

\author{
Bernard Gainot
}

\section{RÉFÉRENCE}

Yves Benot, Marcel Dorigny (dir.), Grégoire et la cause des Noirs (1789 - 1831). Combats et projets, Paris, Publications de la Société française d'histoire d'Outre-mer et de l'Association pour l'étude de la colonisation européenne, 2005, 175 p., ISBN 2-85970-023-4, $16 €$

C'est la deuxième édition d'un volume d'articles consacrés à l'un des engagements les plus constants de l'abbé Grégoire contre la traite négrière, l'esclavage, et le préjugé de couleur, publiés en 2000; ce qui témoigne de l'intérêt pour cette question. La précédente édition a été complétée ici par deux textes importants ; la conférence que l'historien haïtien Duraciné Vaval prononça le 31 mai 1931, à Port-au-Prince, à l'occasion de la commémoration du centenaire de la mort du prélat; et un manuscrit inédit de Grégoire, Observations sur la Constitution du nord d'Haïti, qui est une condamnation sans appel du régime du roi Christophe. Ces documents soulignent à la fois l'aura dont bénéficia la figure de l'abbé dans la première république noire indépendante, l'intérêt manifesté par celui-ci pour les premiers pas de la jeune république, mais aussi les désillusions et les contradictions que firent naitre chez lui cette évolution. Alyssa Goldstein Sepinwall analyse plus précisément ces rapports difficiles (Grégoire et Haïti, un héritage compliqué). Toutes les espérances déçues par l'évolution du cours des affaires dans la France bonapartiste, Grégoire les reporta sur le nouvel État antillais. Il se voyait un peu comme la figure tutélaire de celui-ci, attendant d'une régénération de la société des nouveaux libres la concrétisation des changements auxquels l'ancienne métropole avait renoncé. Non sans ambiguïté, et sans un certain paternalisme, que l'auteur suggère à travers le rapprochement avec le bas-relief de David d'Angers, Les bienfaits de l'imprimerie, sur lequel les Européens apportent les lumières de la civilisation aux « peuples-enfants ». Cette régénération morale et sociale, 
selon Grégoire, doit être intrinsèquement liée à la forme républicaine du gouvernement; aussi la monarchie de Christophe ne pouvait-elle lui apparaître que comme une trahison et une nouvelle tyrannie. Il reporte donc ses espoirs vers la République de Pétion au sud, quitte à en gommer tous les aspects oligarchiques. Mais c'est en définitive le désenchantement qui l'emporte, avec la conclusion de l'épitre aux Haïtiens de 1827 ; « Haïtiens... Adieu ! »

2 Marcel Dorigny retrace l'historique du combat anti-esclavagiste de Grégoire (Grégoire et le combat contre l'esclavage pendant la Révolution), depuis son adhésion à la Société des Amis des noirs, en décembre 1789, sans doute sous l'influence de son ami Lanjuinais. Sans jamais perdre de vue l'objectif ultime de l'abolition, il épouse totalement l'engagement des sociétaires en faveur des libres de couleur, contre l'édifice ségrégationniste de la société antillaise. S'il émit ultérieurement des réserves sur les conditions précipitées dans lesquelles furent pris les décrets d'abolition sous la Convention, jamais il ne les manifesta publiquement ; et il ne cessa jamais de considérer la liberté générale comme un acquis irréversible. En témoigne son implication dans les activités de la Société des Amis des noirs et des colonies sous le Directoire, dont il rédigea les "notes de séance ». Il admet alors pleinement le principe de la continuité territoriale, l'appartenance des territoires coloniaux devenus « départements d'outremer » à la République française. Il estimait certainement que l'évolution ultérieure de ces territoires vers l'indépendance était inéluctable, et il considéra également comme un acquis irréversible la proclamation de janvier 1804. Mais la rupture était alors imputable à la décision du pouvoir consulaire de monter une expédition armée ; tant que la Constitution de l'an III garantissait le principe de l'abolition de l'esclavage, l'appartenance à la République était une protection contre la remise en cause de celleci.

3 Ce qui ne gomme pas toutes les interrogations sur les conceptions véritables de Grégoire en matière de colonisation ; dans une deuxième contribution, Marcel Dorigny s'efforce de clarifier le problème, en évitant soigneusement les écueils de l'apologie béate, mais surtout de la condamnation rétrospective (Intégration républicaine des colonies et projets de colonisation pour l'Afrique : civiliser pour émanciper ?). Il présente tour à tour les deux volets du problème; pour les colonies existantes, dites «colonies modernes ", il faut appliquer le principe de la continuité territoriale, et une législation identique pour tous les territoires de la République. Cette "intégration républicaine ", voulue par la Constitution de l'an III (même si son application effective ne vit en réalité jamais le jour), fut brisée par les initiatives du Consulat. Ne restait alors que l'« indépendance », notion qui recouvrait jusqu'alors la défense d'un régime créole, édifié dans le seul intérêt de la caste blanche; dès lors que cette indépendance est établie au nom de la majorité de la population coloniale, pour la prémunir contre un retour de la servitude, Grégoire s'y rallie sans réserve. Toutefois, le sort de ces «colonies modernes» doit être bien distingué de celui des "colonies nouvelles», établissements à installer sur les territoires africains, qui se substitueraient aux comptoirs de traite. Pas de déportation de la main-d'œuvre, un système économique fondé sur le travail libre, sur l'exemple de la Sierra Leone, dont le projet est repris et popularisé par la seconde Société des Amis des noirs. L'Afrique est ainsi au centre d'un vaste dessein "civilisateur», auquel Grégoire participa par l'intermédiaire de la "Société de l'Afrique intérieure et de la découverte ", créée à Marseille au début de l'année 1800 , et à laquelle Grégoire fut admis en mai 1802. Il trouvait là le moyen de 
prolonger les réflexions sur le projet de "civiliser » l'Afrique pour « émanciper » son peuple, projet de régénération esquissé sous le Directoire ; égalité totale entre noirs et blancs, sans autre distinction que celle du mérite appuyée sur le travail libre et productif, dont la rentabilité serait assurée par des transferts massifs de technologie en provenance d'Europe (les outils de production - charrues, moulins, chariots - étant destinés à remplacer les produits de la traite, spécialement les armes et les denrées ostentatoires). Ce projet est complété par un programme d'éducation technique, sociale, et surtout morale, la dimension religieuse étant la clef de voûte de la réussite du projet.

4 Marcel Dorigny propose, pour caractériser le projet, l'expression de « démarche pédagogique ", qui lui semble à juste titre plus appropriée que celle de "démarche paternaliste ", par laquelle on la stigmatise trop souvent sans la comprendre.

5 C'est dire aussi à quel point ce projet est lié à tous les combats ultérieurs pour la destruction des barrières raciales. Victor Schoelcher s'est appuyé largement sur les acquis du premier mouvement abolitionniste, pour proposer aux territoires qui furent définis comme les «vieilles colonies » un régime d'égalité législative (isonomie) que n'eut certes pas désavoué Grégoire. Anne Girollet, qui a écrit un ouvrage tout à fait passionnant et fondateur sur Victor Schoelcher, présente les six prix proposés au concours dans le testament de Grégoire. Les six questions proposées à la réflexion des candidats, dans une forme héritée de l'académisme des Lumières, couvrent un champ méthodologique extrêmement vaste (L'abbé Grégoire, son legs; six concours pour la liberté et l'égalité) ; la quatrième question porte plus particulièrement sur le problème racial ("Quels seraient les moyens d'extirper le préjugé injuste et barbare des Blancs, contre la couleur des Africains et des sang-mêlé ?»). Schoelcher a concouru avec un mémoire très largement loué par le jury, mais, contrairement à une idée reçue, ce n'est pas ce mémoire qui a été primé, en raison, nous dit Anne Girollet, de son attention trop exclusivement portée vers la question des moyens de l'abolition, plutôt que de se limiter au cadre défini par la question posée ; les relations raciales. C'est S. Linstant, de Haïti, qui fut lauréat, en développant des thèmes qui s'inscrivent tout à fait dans la continuité des solutions proposées par Grégoire, insistant, comme ce dernier, sur le fait que le préjugé de couleur ne s'éteint pas de lui-même avec l'abolition ; insistant aussi sur l'instruction et l'implantation d'écoles élémentaires mixtes, l'égalité d'accès aux emplois publics, l'incitation aux mariages mixtes, le développement des caisses d'épargne et du crédit public.

6 Ces solutions, Grégoire les développa inlassablement, au cours de la décennie révolutionnaire, et plus particulièrement sous le Directoire, certes, mais aussi à l'époque de la Restauration. Et le grand mérite de ce volume est de mettre l'accent sur cette période, trop négligée, mais pourtant cruciale pour le passage de relais à la génération de 1848. Yves Benot a étudié les articles de Grégoire dans les revues de la période de la Restauration (Grégoire défenseur de la cause des noirs dans les revues de la Restauration) : la Chronique religieuse, qu'il co-dirige avec Lanjuinais, de 1819 à 1822 ; la Revue Encyclopédique, où il donne de nombreux articles à son ami Marc-Antoine Jullien, de 1822 à 1827. Dans la première, qui milite essentiellement en faveur du catholicisme gallican, il dénonce la traite négrière illicite, et il analyse longuement la politique religieuse de Haïti. Dans la seconde, il met en garde contre les espions envoyés d'Europe, et particulièrement de Rome, dans la république antillaise; et, toujours, il dénonce la traite illicite et la perpétuation de l'esclavage, en s'appuyant notamment sur 
les écrits du puissant mouvement abolitionniste anglais, dont il salue la persévérance face à la tiédeur des autres nations. Il cherche également à mettre en valeur les ouvrages des intellectuels noirs et mulâtres, dans la ligne de son ouvrage de 1808, De la littérature des nègres, dont rend compte Lucien-René Abénon. L'auteur analyse précisément le contexte dans lequel cet ouvrage a vu le jour, en pleine régression colonialiste et raciste de la politique officielle de l'Empire, mais il en souligne également les limites; par " littérature des nègres ", il met l'accent sur des récits et des poèmes de valeur inégale, privilégiant notamment les récits de vies d'esclaves, qui avaient déjà connu un certain succès dans l'Europe de la fin du XVIII ${ }^{\mathrm{e}}$ siècle, avec une certaine nostalgie d'une époque où l'opinion publique était bien plus réceptive aux arguments abolitionnistes. Mais il ignore les cosmogonies africaines et les récits de la tradition orale. En dépit de ce caractère nostalgique et de ses limites, l'ouvrage attira des réponses indignées, comme Le cri des colons de Richard de Tussac, qui avaient bien perçu l'essentiel de la démonstration; en insistant sur la littérature comme quintessence de la civilisation, il s'agissait bel et bien de démontrer que la race noire n'était pas essentiellement vouée à la sauvagerie.

7 Et c'est bien la raison de l'aura dont bénéficie encore l'abbé dans l'Afrique indépendante. Amady Aly Dieng analyse justement la portée de La littérature des nègres pour l'historiographie africaine de ces cinquante dernières années (L'abbé Grégoire et l'Afrique noire aujourd'hui). Il fait une présentation des auteurs, noirs et mulâtres, recensés par Grégoire, et il confronte les hypothèses avancées par Grégoire sur les origines de la civilisation africaine, aux travaux les plus récents des historiens africains sur la question, qui confortent sur bien des points les intuitions de l'abbé.

Le combat de celui qui voulut être jusqu'à la fin l'évêque de Blois ne peut, toutefois, pas se comprendre si on le sépare de son fondement moral, et notamment de la perspective apologétique de la conversion de l'humanité au christianisme. Le rapprochement avec une autre grande figure chrétienne de la lutte contre la domination coloniale, Barthélémy de Las Casas, l'évêque du Chiapas, s'impose d'autant plus que Grégoire a consacré toute une étude à son illustre prédécesseur, Apologie de Las Casas, en 1800, dont Bernard Plongeron nous rappelle la genèse. Mais l'étude va bien au-delà du contexte ; c'est à une réhabilitation de l'évêque du Chiapas que s'attache Grégoire, s'attaquant à l'interprétation fallacieuse selon laquelle Las Casas aurait préconisé la mise en esclavage des noirs en lieu et place des Indiens susceptibles d'être convertis au christianisme. Pour mieux dégager la portée de cette réhabilitation, l'auteur se livre à un rappel passionnant des sources historiques et théologiques de l'action du prélat espagnol.

9 Les fondements chrétiens de l'abolitionnisme de Grégoire sont étudiés par Rita Hermon-Belot. C'est une conception de l'unité de la famille humaine qui sous-tend tous les écrits de Grégoire entre 1789 et 1797, que reprend l'auteur. Seule une république chrétienne peut porter à son terme un authentique projet émancipateur. Si la désillusion envers le facteur politique est croissante à partir de 1797, ce n'est que pour mieux insister sur le caractère universaliste du message chrétien. Ann Thomson rappelle tout particulièrement les doctrines différentialistes auxquelles l'abbé fut confronté, et qu'il réfuta sans relâche (Grégoire et l'unité de l'espèce humaine) : doctrines qui prenaient appui en particulier sur l'œuvre taxinomique de Linné, pour en tirer des conclusions sur le classement hiérarchique des espèces humaines, à l'issue desquelles le noir était toujours placé tout en bas de l'échelle, et fréquemment rejeté du côté de 
l'animalité (Blumenbach, Malte-Brun, Bory de Saint-Vincent). Grégoire est un adepte du monogénisme, contre toutes ces théories polygénistes qui fondent le racisme «scientifique » du XIX ${ }^{e}$ siècle. L'analyse de la controverse sur cette question dans le monde anglo-saxon est tout à fait éclairante, et l'auteur souligne que Grégoire était parfaitement au fait de la dite controverse.

10 Cette contribution, qui ouvre le volume, a le mérite de faire ressortir la cohérence et la profondeur du projet « régénérateur » de Grégoire, qui sous-tend l'ensemble des autres contributions, dont le grand mérite est de nous restituer l'ensemble du parcours de l'abbé, dont ces années de la Restauration, trop souvent négligées.

11 Un ouvrage extrêmement novateur, donc, qui ouvre des pistes de recherche sur les multiples facettes de la pensée et de l'action de cette grande figure historique. 